\title{
Esophageal adenosquamous carcinoma mimicking acantholytic squamous cell carcinoma
}

\author{
SUSUMU MATSUKUMA ${ }^{1,2}$, OH TAKAHASHI ${ }^{1}$, YOSHITAKA UTSUMI ${ }^{1}$, MASAKI TSUDA ${ }^{1}$, \\ KOSUKE MIYAI $^{1}$, KENJI OKADA ${ }^{1}$ and HIROAKI TAKEO ${ }^{1}$ \\ ${ }^{1}$ Department of Pathology and ${ }^{2}$ Health Care Center, Japan Self-Defense Forces Central Hospital, \\ Setagaya, Tokyo 154-8532, Japan
}

Received April 22, 2017; Accepted July 27, 2017

DOI: $10.3892 / 01.2017 .6804$

\begin{abstract}
Herein is described a unique case of esophageal cancer mimicking acantholytic squamous cell carcinoma (SCC). The patient succumbed to the disease within one month of diagnosis. Autopsy revealed a $10-\mathrm{cm}$ esophageal tumor, characterized by prominent acantholysis-like areas composed of discohesive cancer cells, along with nested growth of SCC. These discohesive cancer cells focally exhibited pagetoid extension into adjacent esophageal epithelium, comprised $\sim 60 \%$ of the esophageal tumor volume and had widely metastasized to the lungs, chest wall, liver, spleen, right adrenal gland, bones and lymph nodes. No metastases of SCC were observed. SCC cells were immunohistochemically positive for keratin 5/6 and E-cadherin and were negative for mucin and carcinoembryonic antigen (CEA). However, the discohesive cancer cells exhibited negativity for keratin $5 / 6$, positivity for mucin and CEA, and diminished or no immunostaining for E-cadherin. Thus, these discohesive cells represented true adenocarcinomatous differentiation rather than acantholytic SCC cells. It was concluded that this tumor was an esophageal adenosquamous carcinoma with 'pseudo'-acantholytic adenocarcinoma components, which should be considered as a rare but distinctive type of aggressive cancer.
\end{abstract}

\section{Introduction}

Squamous cell carcinoma (SCC) is the most common type of esophageal cancer, followed by adenocarcinoma; these two

Correspondence to: Dr Susumu Matsukuma, Department of Pathology, Japan Self-Defense Forces Central Hospital, 1-2-24 Ikejiri, Setagaya-ku, Tokyo 154-8532, Japan

E-mail: skuma@cocoa.plala.or.jp

Abbreviations: CEA, carcinoembryonic antigen; di-PAS, periodic acid-Schiff stain after diastase digestion; K5/6, keratin 5/6; EMA, epithelial membrane antigen; H\&E, hematoxylin and eosin; SCC, squamous cell carcinoma

Key words: acantholytic SCC, adenosquamous carcinoma, esophagus, E-cadherin, adenoid SCC, CEA histological types account for $>90 \%$ of primary esophageal cancer cases (1-5). Adenocarcinoma arises chiefly in Barrett mucosa and rarely from ectopic gastric mucosa or esophageal glands (2). However, focal adenocarcinomatous differentiation occurs in $20 \%$ of cases of esophageal invasive SCC $(1,6)$. When the adenocarcinomatous features occupy considerable amounts of the esophageal SCC, tumors are classified as either adenosquamous carcinoma or mucoepidermoid carcinoma (1,2,7-11). Adenocarcinomatous differentiation in SCC should be distinguished from acantholytic SCC (also called pseudoglandular or adenoid SCC) (12-19). Here was encountered a unique autopsy case of esophageal adenosquamous carcinoma mimicking acantholytic SCC, and herein is describe the clinicopathological findings of this case.

\section{Case report}

A 53-year-old Japanese male was hospitalized firstly to Japan Self-Defense Force Hanshin Hospital (Hyogo, Japan) on April 1, 2011, and subsequently to Japan Self-Defense Forces Central Hospital (Tokyo, Japan) on April 14, 2011, for evaluation of fever and hoarseness. Laboratory examination revealed increased serum levels of C-reactive protein and carcinoembryonic antigen (CEA). Endoscopic examination disclosed an ulcerating esophageal tumor. The biopsy specimen exhibited features of moderate-poorly differentiated SCC. A computed tomography scan confirmed the presence of an esophageal tumor and revealed possible metastatic nodules in the liver and mediastinal and intra-abdominal lymph nodes (Fig. 1). The fever and hoarseness were considered to be caused by tumor necrosis and lymph node metastasis-associated recurrent nerve palsy, respectively. The patient rapidly deteriorated and succumbed to respiratory insufficiency one month following first hospitalization.

Autopsy revealed a $10-\mathrm{cm}$ ulcerating esophageal tumor (Fig. 2A) directly invading the trachea. Microscopically, this tumor partially exhibited nested proliferation composed of polygonal and/or short spindle cells containing swollen pleomorphic nuclei without distinct keratinization (Fig. 2B). These features indicate a diagnosis of poor-moderately differentiated SCC. However, invading cancerous nests frequently exhibited geographic acantholysis-like changes (Fig. 2C), chiefly composed of poorly cohesive, monomorphic neoplastic 
cells with relatively scant cytoplasm. These acantholytic areas were slightly demarcated, occasionally contained necrotic cells (Fig. 2D), and lacked columnar cells, intermediate cells and neoplastic cells forming tubules or intracytoplasmic lumina. These discohesive cancer cells also invaded in an alveolar fashion (Fig. 2E), reminiscent of micropapillary carcinoma, and focally involved the adjacent esophageal epithelium in a pagetoid manner (Fig. 2F). In total, these discohesive cancer cells comprised $\sim 60 \%$ of the tumor volume, and had widely metastasized to the lungs, chest wall, liver, spleen, right adrenal gland, vertebral bones and generalized lymph nodes with multifocal necrosis. Notably, in both lungs, metastatic discohesive cells exhibited prominent lymphangiosis (Fig. 2G), which would have contributed to the respiratory failure and causing the patient's mortality. Metastatic SCC cells were not observed.

In the primary esophageal tumor, SCC cells were negative for mucin staining and CEA and were weakly positive for keratin 5/6 (K5/6); scattered positivity for epithelial membrane antigen (EMA) in SCC cells was also observed. By contrast, discohesive cancer cells commonly expressed mucin, which was detected by alcian blue and/or periodic acid-Schiff (PAS) staining with or without diastase digestion (Fig. 3A and F). In addition, discohesive cancer cells exhibited strong positivity for EMA (Fig. 3B) and CEA (Fig. 3C), and negativity for K5/6 (Fig. 3D). In the primary tumor, SCC cells exhibited
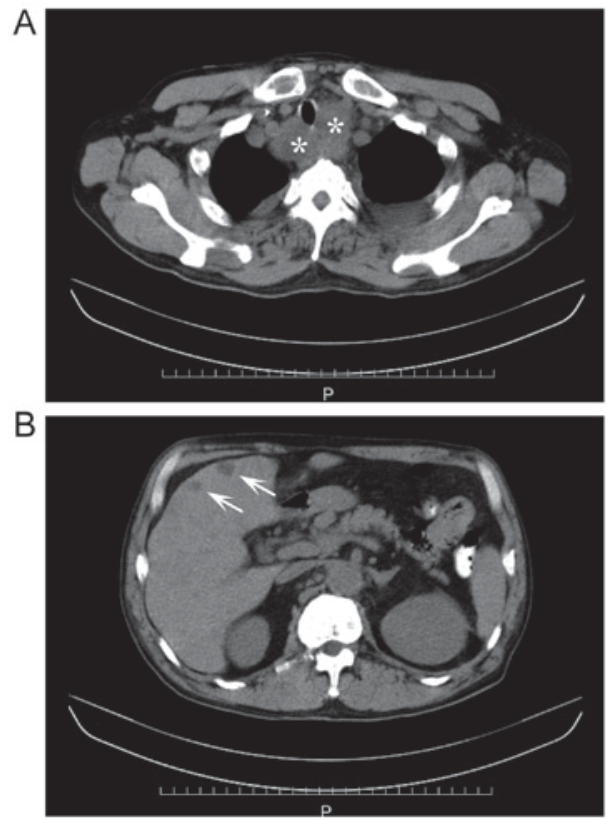

Figure 1. Computed tomography scanning demonstrates swollen mediastinal lymph nodes (A, asterisks) and liver nodules (B, arrows).

weak cytoplasmic positivity for E-cadherin. By contrast, discohesive cancer cells exhibited reduced or no E-cadherin
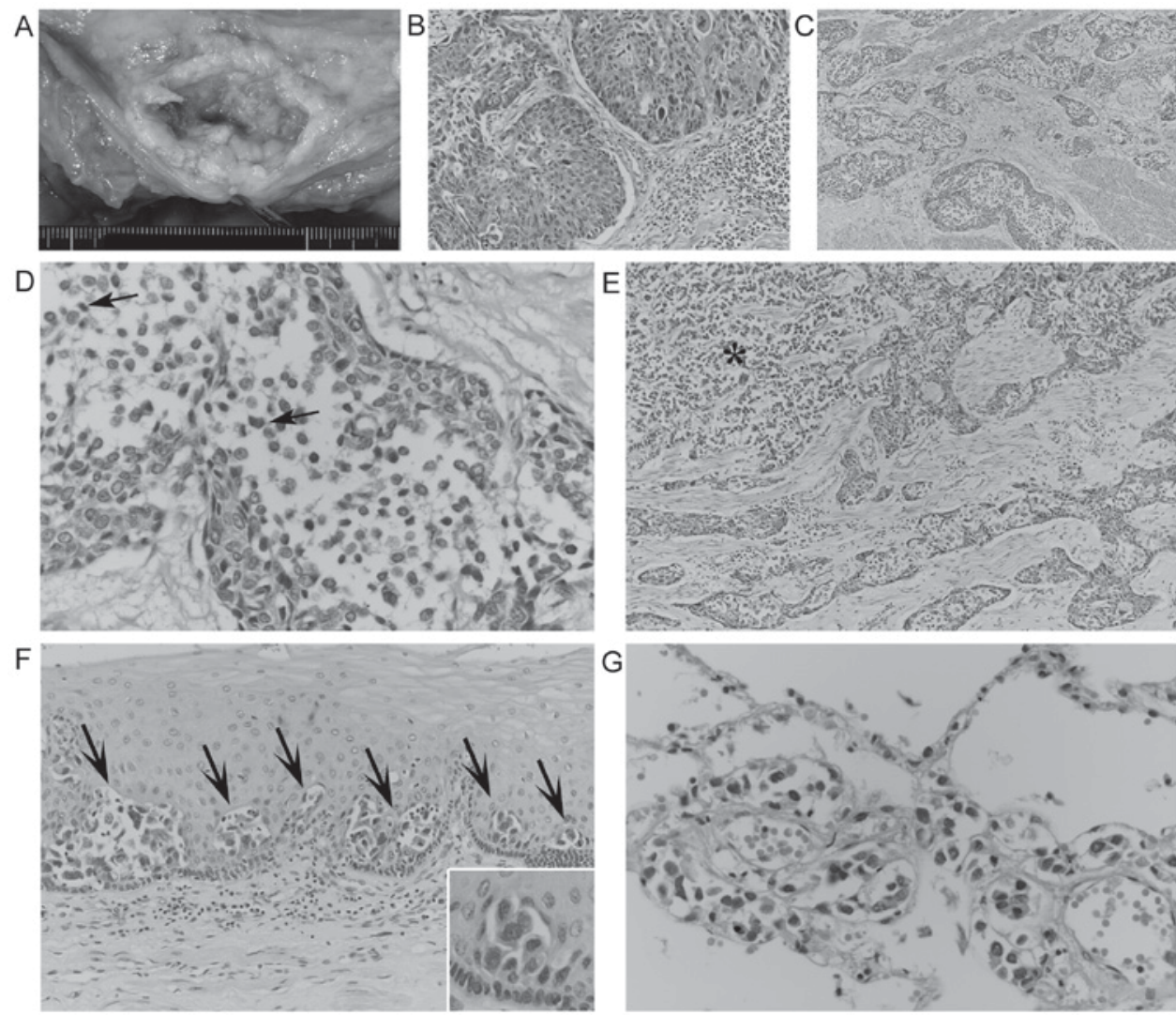

Figure 2. Gross appearance and hematoxylin and eosin-stained histology of the esophageal tumor and metastases. (A) Gross features of the ulcerating esophageal tumor. (B) Solid nested growth of squamous cell carcinoma (magnification, x200). (C) Invading cancerous nests with prominent acantholysis-like areas (magnification, x100). (D) High-power view (x400) of acantholysis-like areas composed of discohesive cancer cells with occasional necrotic cells (arrows; magnification, x400). (E) Alveolar cancerous proliferation (asterisk) near nested growth with acantholysis-like changes (magnification, x100). (F) Pagetoid extension of discohesive cancer cells in the esophageal epithelium (magnification, x200) and a high-power view (inset, x400) of pagetoid cells. (G) Pulmonary lymphangiosis of metastatic discohesive cancer cells (magnification, $\mathrm{x} 400$ ). 

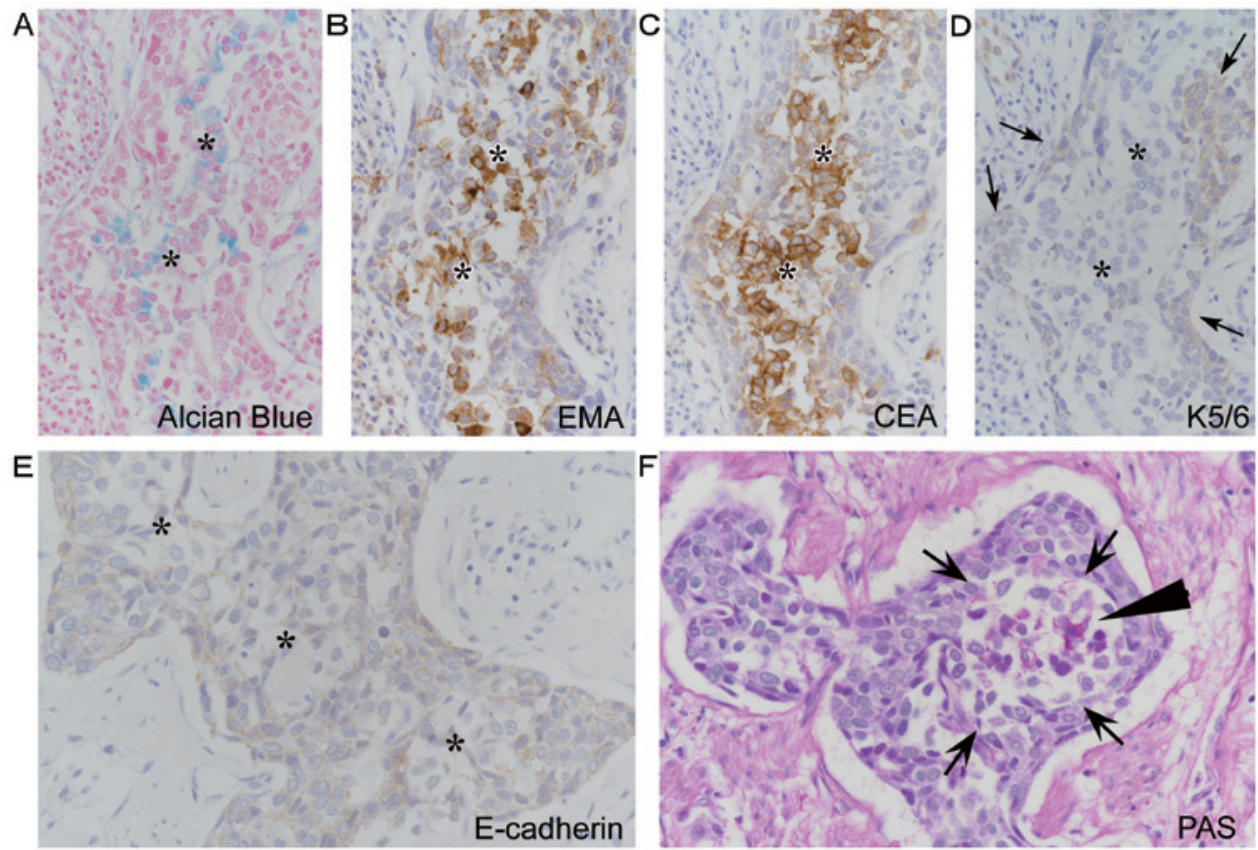

Figure 3. Histochemical and immunohistochemical findings of the primary esophageal cancer. (A-D) In the 'pseudo'-acantholytic areas (asterisks) of the primary tumor, discohesive cancer cells exhibit (A) alcian blue positive mucin, and (B) strong positivity for EMA (C) and CEA, and no staining for K5/6. By contrast, the surrounding cohesive squamoid cells exhibit no staining for CEA (C) and weak positivity for K5/6 (D, arrows; magnification, x400). (E) Diminished E-cadherin staining of discohesive cells (asterisks) in the acantholysis-like area of the primary tumor, compared with the weak positivity of the surrounding cohesive squamoid cells (magnification, x400). (F) Certain cancer cells (arrowhead) within the 'pseudo'-acantholytic area (arrows) have PAS stain-positive mucin (magnification, x400). CEA, carcinoembryonic antigen; EMA, epithelial membrane antigen; K5/6, keratin 5/6; PAS, periodic acid-Schiff.
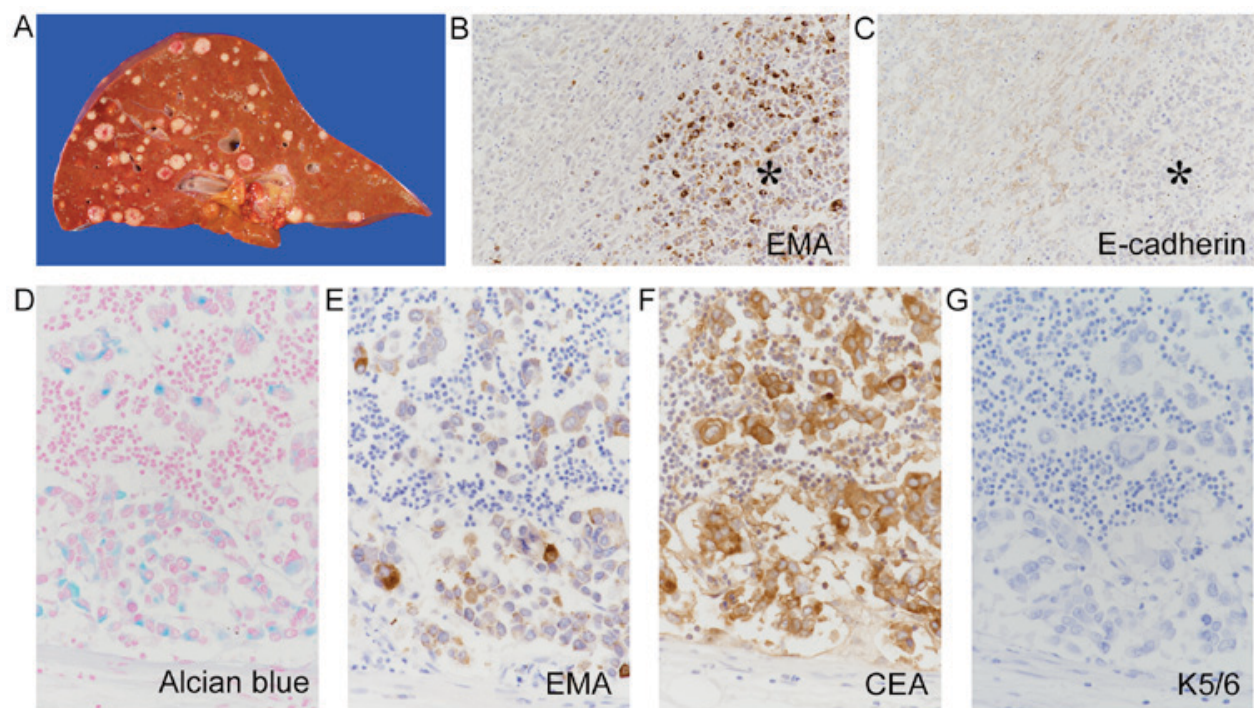

Figure 4. Pathological findings of the metastatic lesions. (A) Gross features of multiple metastatic nodules in the liver. (B and C) Metastatic liver nodules composed of discohesive cancer cells (asterisk) exhibit positivity for EMA (B) and little or no staining for E-cadherin (C) (B and C; magnification, x200). (D and E) Metastatic cancer cells in the abdominal para-aortic lymph node exhibit (D) alcian blue-positive mucin, (E) EMA positivity, (F) CEA positivity and (G) K5/6 negativity (D-G, magnification, x400). CEA, carcinoembryonic antigen; EMA, epithelial membrane antigen; K5/6, keratin 5/6.

immunostaining as compared with the weak staining of the surrounding cohesive cancer cells (Fig. 3E). In metastatic lesions, cancer cells exhibited occasional expression of mucin, and EMA positivity, CEA positivity and K5/6 negativity were observed via immunohistochemistry (Fig. 4). The majority of metastatic cancer cells exhibited negative or reduced E-cadherin immunostaining (Fig. 4C), although a minority of metastatic cancer cells were positive for E-cadherin. No positivity for $\mathrm{p} 63, \alpha$-fetoprotein, placental alkaline phosphatase or vimentin was observed in any of the primary or metastatic cancerous lesions.

\section{Discussion}

The present esophageal tumor exhibited not only nested growth of SCC, but also frequent, loosely arranged acantholysis-like 
areas, which occasionally contained necrotic cells and lacked columnar cells and neoplastic tubules. These features closely mimicked acantholytic SCC, which is an uncommon but distinctive variant of SCC with dyskeratosis-associated acantholytic changes $(3,13-18)$. However, in the present acantholysis-like areas, the discohesive cancer cells exhibited alcian blue+ and/or PAS+ mucin, and were strongly positive for EMA and CEA. No K5/6 positivity was observed in these discohesive cells; however, K5/6 positivity was scattered in the SCC components and cohesive cells around the acantholysis-areas. These findings indicate that these acantholysis-like areas represent true adenocarcinomatous differentiation rather than acantholytic changes of SCC. The esophageal tumor involved both these adenocarcinoma (60\%) and SCC (40\%) cells, and lacked intermediate cells suggestive of mucoepidermoid carcinoma $(1,2,7)$. Therefore, it was concluded that this tumor was esophageal adenosquamous carcinoma with 'pseudo'-acantholytic adenocarcinoma components.

In the present case, the alveolar growth of the discohesive adenocarcinoma cells focally resembled those of micropapillary carcinoma in other sites (20-22). However, the present alveolar cancer cells formed no distinctive micropapillary tufts or aggregations with a reversed polarity, eliminating a possible diagnosis of micropapillary carcinomatous components. Alveolar or prominently discohesive changes of adenocarcinoma cells have been rarely described in the lung (23), but these cases had no SCC components. Alwaheeb and Chetty (24) reported a case of acantholytic adenosquamous carcinoma of the pancreas. However, these acantholytic changes were observed in the SCC components, and not in the adenocarcinomatous components. Lee (19) described 'signet ring cells' in a case of esophageal acantholytic SCC, but these were negative for mucin and were considered to have arisen from vacuolar changes. The present review of the literature failed to reveal any cases of adenosquamous carcinoma with 'pseudo'-acantholytic adenocarcinoma components in the esophagus or other sites.

Acantholytic changes of SCC are known to be associated with reduced E-cadherin immunoreactivity $(14,15)$. In the present case, the discohesive adenocarcinoma cells exhibited diminished or negative staining for E-cadherin despite weak positivity in SCC cells. Furthermore, the discohesive adenocarcinoma cells aggressively metastasized to several organs, directly contributing to the patient's mortality, although SCC cells were not observed in the metastatic lesions. Furthermore, the majority of the metastatic cancer cells exhibited little or no E-cadherin staining. These findings suggest that the loss or alteration of E-cadherin in adenocarcinoma cells causes 'pseudo'-acantholysis and also serves a role in aggressive metastasis (25). Notably, the adenocarcinoma cells in the present case focally involved the adjacent esophageal epithelium in a pagetoid manner, which is exceptionally rare in the esophagus (26,27). Abraham et al (27) also noted similar diminished E-cadherin immunostaining in esophageal Paget cells.

In conclusion, herein is described an autopsy case of esophageal adenosquamous carcinoma with 'pseudo'-acantholytic adenocarcinoma components, possibly associated with the loss of E-cadherin. Therefore, this tumor should be considered as a rare but aggressive type of cancer in the differential diagnoses of esophageal neoplasms.

\section{References}

1. Lewin KJ and Appelman HD: Tumors of the esophagus and stomach. In: Atlas of Tumor Pathology. 3rd edition. Fascicle 18. Rosai J and Sobin LH (eds). American Registry of Pathology, Washington, DC, pp43-144, 1996.

2. Bosman FT, Carneiro F, Hruban RH and Theise ND (eds): WHO Classification of Tumours of the Digestive System. 4th edition. International Agency for Research on Cancer, Lyon, pp18-31, 2010.

3. Enzinger PC and Mayer RJ: Esophageal cancer. N Engl J Med 349: 2241-2252, 2003.

4. Brown LM, Devesa SS and Chow WH: Incidence of adenocarcinoma of the esophagus among white Americans by sex, stage, and age. J Natl Cancer Inst 100: 1184-1187, 2008.

5. Yang CS, Chen X and Tu S: Etiology and prevention of esophageal cancer. Gastrointest Tumors 3: 3-16, 2016.

6. Takubo K, Sasajima K, Yamashita K, Tanaka Y, Fujita K, Mafune K and Wang QH: Morphological heterogeneity of esophageal carcinoma. Acta Pathol Jpn 39: 180-189, 1989.

7. Lam KY, Loke SL and Ma LT: Histochemistry of mucin secreting components in mucoepidermoid and adenosquamous carcinoma of the oesophagus. J Clin Pathol 46: 1011-1015, 1993.

8. BombíJA, Riverola A,Bordas JM and Cardesa A: Adenosquamous carcinoma of the esophagus. A case report. Pathol Res Pract 187: 514-521, 1991.

9. Yachida S, Nakanishi Y, Shimoda T, Nimura S, Igaki H, Tachimori $\mathrm{Y}$ and Kato $\mathrm{H}$ : Adenosquamous carcinoma of the esophagus. Clinicopathologic study of 18 cases. Oncology 66: 218-225, 2004.

10. Chen SB, Weng HR, Wang G, Yang JS, Yang WP, Liu DT, Chen YP and Zhang H: Primary adenosquamous carcinoma of the esophagus. World J Gastroenterol 19: 8382-8390, 2013.

11. Ni PZ, Yang YS, Hu WP, Wang WP, Yuan Y and Chen LQ: Primary adenosquamous carcinoma of the esophagus: An analysis of 39 cases. J Thorac Dis 8: 2689-2696, 2016.

12. Cardesa A, Zidar N and Alos L: Acantholytic squamous cell carcinoma. In: WHO Classification of Tumours. Pathology and Genetics of Head and Neck Tumours. Barnes L, Eveson JW, Reichart P and Sidransky D (eds). IARC press, Lyon, pp129, 2005.

13. Elder DE, Elenitsas R, Rosenbach M, Murphy GF, Rubin AI and Xu X (eds): Lever's Histopathology of the Skin. 11th edition. Wolters Kluwer, Philaderphia, PA, pp1003, 2015.

14. Zidar N, Gale N, Župevc A and Dovšak D: Pseudovascular adenoid squamous-cell carcinoma of the oral cavity-a report of two cases. J Clin Pathol 59: 1206-1208, 2006.

15. Gu X, Jiang R and Fowler MR: Acantholytic squamous cell carcinoma in upper aerodigestive tract: Histopathology, immunohistochemical profile and epithelial mesenchymal transition phenotype change. Head Neck Pathol 6: 438-444, 2012.

16. Terada T: Adenoid squamous cell carcinoma of the oral cavity. Int J Exp Pathol 5: 442-447, 2012.

17. Cunha IW, Guimaraes GC, Soares F, Velazquez E, Torres JJ, Chaux A, Ayala G and Cubilla AL: Pseudoglandular (adenoid, acantholytic) penile squamous cell carcinoma: A clinicopathologic and outcome study of 7 patients. Am J Surg Pathol 33: $551-555,2009$.

18. Shah AA, Jeffus SK and Stelow EB: Squamous cell carcinoma variants of the upper aerodigestive tract: A comprehensive review with a focus on genetic alterations. Arch Pathol Lab Med 138: 731-744, 2014.

19. Lee D: Acantholytic squamous cell carcinoma of the esophagus with prevalent single isolated tumour cells including signet ring cells and many osteoclast-like giant cells. Pathology 48: 281-283, 2016.

20. Luna-Moré S, Gonzalez B, Acedo C, Rodrigo I and Luna C: Invasive micropapillary carcinoma of the breast. A new special type of invasive mammary carcinoma. Path Res Pract 190: 668-674, 1994.

21. Sánchez-Mora N, Presmanes MC, Monroy V, Moreno N, Lara-Martínez JM, Aladro MH and Álvarez-Fernández E: Micropapillary lung adenocarcinoma: A distinctive histologic subtype with prognostic significance. Case series. Hum Pathol 39: 324-330, 2008. 
22. Reis-Filho JS and Ellis IO: Invasive micropapillary carcinoma. In: WHO Classification of Tumours of the Breast. 4th edition. Lakhani SR, Ellis IO, Schnitt SJ, Tan PH and van de Vijver MJ (eds). International Agency for Research on Cancer, Lyon, pp65-66, 2012.

23. Kozu Y, Isaka M, Ohde Y and Nakajima T: Aggressive adenocarcinoma of the lung consisting solely of discohesive cells. J Cardiothoracic Surg 8: 89, 2013.

24. Alwaheeb S and Chetty R: Adenosquamous carcinoma of the pancreas with an acantholytic pattern together with osteoclast-like and pleomorphic giant cells. J Clin Pathol 58: 987-990, 2005 .
25. Shiozaki H, Tahara H, Oka H, Miyata M, Kobayashi K, Tamura S, Iihara K, Doki Y, Hirano S, Takeichi M, et al: Expression of immunoreactive E-cadherin adhesion molecules in human cancers. Am J Pathol 139: 17-23, 1991.

26. Matsukuma S, Aida S, Shima S and Tamai S: Paget's disease of the esophagus. A case report with review of the literature. Am J Surg Pathol 19: 948-955, 1995.

27. Abraham SC, Wang H, Wang KK and Wu TT: Paget cells in the esophagus: Assessment of their histopathologic features and near-universal association with underlying esophageal adenocarcinoma. Am J Surg Pathol 32: 1068-1074, 2008. 